\section{Mein Schmerz ist dein Schmerz}

Einer dänischen Studie zufolge beeinflusst der Gesundheitszustand der Mutter offenbar deren Fähigkeit, Schmerzen bei ihrem Kind objektiv zu beurteilen. Mütter, die unter chronischen Schmerzen litten, berichteten fünfmal so häufig über rezidivierende Schmerzzustände ihrer Kinder wie Mütter, die selbst schmerzfrei waren, so die Ergebnisse der fragebogenbasierten Querschnittstudie. Teilgenommen haben 131 gesunde Kinder im Alter zwischen sechs und elf Jahren sowie deren Mütter.

Graungaard AH et al. Acta Paediatrica 2015 (online first)

\section{Zappeln gesünder als Stillsitzen?}

Wer viel sitzt, erwirbt zahlreiche Gesundheitsrisiken: Die Wahrscheinlichkeit für Diabetes, kardiovaskuläre Erkrankungen und Tod ist Studien zufolge signifikant höher. Für Vielsitzer bietet eine britische Studie eine mögliche Lösung: Frauen, die sich in sitzender Position besonders viel bewegten, hatten bei einer täglichen Sitzdauer von 5-6 Stunden sogar ein um 37\% geringeres Sterberisiko als bei kürzerer Sitzzeit. An der Studie waren fast 13.000 Frauen im Alter zwischen 37 und 78 Jahren beteiligt.

Hagger-Johnson G et al. Am J Prev Med 2015 (online first)

\section{Ärztinnen verdienen deutlich weniger}

\begin{abstract}
Dass Frauen trotz gleicher Qualifikation in Angestelltenverhältnissen oft weniger als ihre männlichen Kollegen verdienen, ist bekannt. Aber auch selbstständig tätige Ärztinnen hinken ihren männlichen Kollegen hinterher. Bei den Hausärzten beträgt die Differenz zwischen Praxisinhaber und -inhaberin im Jahr 42.700€: Frauen arbeiteten weniger Stunden im Jahr, behandelten weniger Privatpatienten und rechneten weniger Leistungen ab, so die Studie.
\end{abstract}

www.aerztezeitung.de

SEER-Registerdaten

\title{
Brustkrebs: Verbessertes Überleben im Spätstadium
}

Manche jüngeren Brustkrebspatientinnen im Stadium IV profitieren möglicherweise von einer Operation als Ersttherapie, und zwar unabhängig von der Tumorgröße. Sie leben länger als Patientinnen ohne Operation, wie die Analyse von Befunden in den USA vermuten lässt, die in einem Zeitraum von mehr als zwei Dekaden erhoben wurden.

Um den Zusammenhang zwischen Operation und Überleben bei Brustkrebspatientinnen im metastasierten Spätstadium zu analysieren, werteten Wissenschaftler die Daten des US-Registers SEER (Surveillance, Epidemiology, and End Results) aus, die zwischen 1988 und 2011 dokumentiert worden waren. Für die Analyse standen die
Befunde von 21.372 Patientinnen zur Verfügung, von denen 8330 Frauen operiert worden waren. Das mediane Überleben lag der Auswertung zufolge bei Frauen, deren Karzinom zwischen 1988 und 1991 entdeckt worden war, bei 20 Monaten und war damit sechs Monate kürzer als bei Frauen mit der Krebsdiagnose zwischen 2007 und 2011. Auch wenn im Vergleich zum Studienbeginn im Verlauf immer seltener operiert wurde, lag die Wahrscheinlichkeit für ein längeres Überleben nach der Operation um $40 \%$ höher. Insgesamt betrachtet lebten Patientinnen ohne Operation als Erstmaßnahme median noch 19 Monate, mit Operation dagegen noch 28 Monate, und zwar unabhängig von der Tumorgröße.

Thomas A et al. JAMA Surg 2015 (online first)

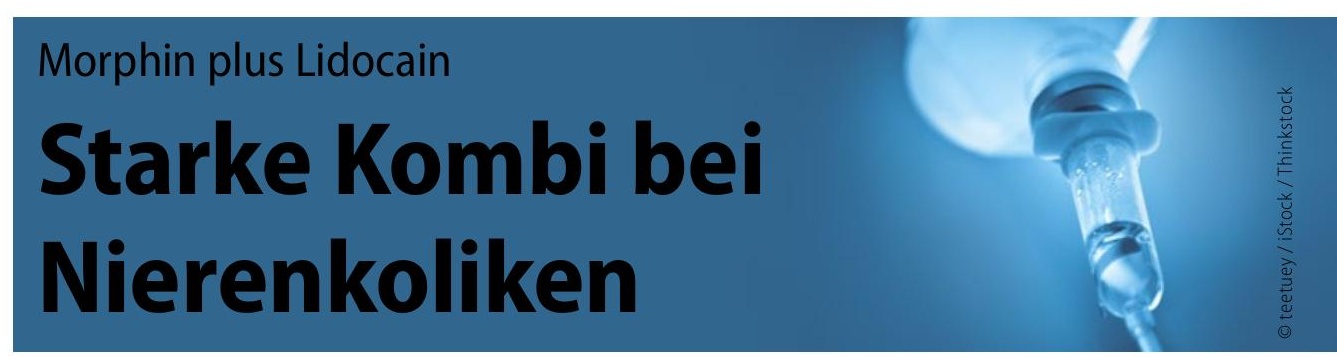

Der extreme Schmerz einer Nierenkolik erfordert eine rasche und effektive Schmerzlinderung, etwa mit Morphin. Kombiniert mit Lidocain ist die Wirkung noch besser.

Um die Dosis und das Nebenwirkungsrisiko so gering wie möglich halten zu können, gleichzeitig aber keine Abstriche in der Schmerzlinderung hinnehmen zu müssen, haben iranische Anästhesisten Morphin mit Lidocain kombiniert und das analgetische Potenzial mit dem einer alleinigen Morphintherapie verglichen. Überprüft haben sie das Therapieregime mit insgesamt 89 Erwachsenen, die mit Nierenkoliken vorstellig wurden. Gruppe A behandelten sie mit $0,1 \mathrm{mg} / \mathrm{kg}$ Morphin plus $1,5 \mathrm{mg} / \mathrm{kg}$ Lidocain, Gruppe B mit $0,1 \mathrm{mg} / \mathrm{kg}$ Morphin plus 9\%-iger
Kochsalzlösung. Vor Infusion sowie 5, 10, 30, 60 und 120 Minuten nach Behandlung sollten die Probanden die Schmerzintensität anhand einer visuellen Analogskala von 0 bis 10 beurteilen, wobei 0 für schmerzfrei stand und 10 für die schlimmsten vorstellbaren Schmerzen. In beiden Gruppen sanken im Zeitverlauf beide Scores signifikant. Dabei waren die Patienten mit der kombinierten Analgesie im Durchschnitt 13 Minuten eher schmerzfrei. Außerdem waren Patienten, die zusätzlich Lidocain erhalten hatten, schon nach median 26,6 Minuten ihre Übelkeit los, die allein mit Morphin Behandelten mussten hingegen median 58,33 Minuten warten.

(dk)

Firouzian A et al. Am J Emerg Med 2015, online 19. November; doi: 10.1016/j.ajem.2015.11.062 